\title{
SHORT STUDIES
}

\section{DAS MESSIASGEHEIMNIS DES MARKUSEVANGELIUMS ${ }^{1}$}

Zur Eigenart des Markusevangeliums gehören zwei fundamentale Tatsachen: Das Markusevangelium ist erstens, weit mehr als die übrigen Synoptiker, eine Passionsgeschichte mit verlängerter Einleitung. Es ist zweitens geprägt durch das sogenannte Messiasgeheimnis, gekennzeichnet vor allem durch die Schweigegebote Jesu gegenüber den Dämonen, die ihn als Gottessohn bezeichnen, durch das Unverständnis der Jünger, die den Leidens- und Auferstehungsankündigungen ratlos gegenüber stehen und durch die besondere Gestaltung der Gleichnisse.

Der Passionscharakter und das Messiasgeheimnis durchdringen und bedingen sich gegenseitig, wie sich vor allem an den Leidensweissagungen zeigt, die den Passions- mit dem Einleitungsteil verklammern.

Es sind drei Gesichtspunkte heraus zu stellen, die beachtet werden müssen, wenn der Skopus des ganzen Evangeliums klar heraustreten soll: (I) Die Methodik der Darstellung des Messiasgeheimnisses; (2) der Inhalt dieses Geheimnisses; (3) der erkenntnismäßige Zugang zu ihm, d. h. die Frage seiner Offenbarung.

Wir greifen jeweils beispielhaft einige augenfällige Texte heraus, um von da aus die Brücke zu anderen Stellen zu schlagen. Dabei ist es selbstverständlich, daß wir auch die Stimmen der durch Markus aufgenommenen Traditionen beachten. Die form-, traditions- und redaktionsgeschichtliche Arbeit darf nicht übergangen werden. Dennoch werden wir nur im Vorbeigehen auf diese Fragen eintreten. Denn es liegt uns vor allem daran, den vorliegenden Text mit seinen Spannungen, Absichten und Grundpositionen zur Geltung zu bringen.

\section{DIE METHODE DER DARSTELLUNG}

Um die Darstellungsmethode des Markus zu durchschauen, gehen wir von dem Verhör und der Verurteilung Jesu durch den Hohen Rat (xiv. 55-65) aus. Dabei ist nicht auf die umstrittenen rechtlichen und historischen Probleme einzutreten, die sich mit dem Prozeß Jesu verbinden. Wir konzentrieren uns auf die Frage: Welche Absicht führt Markus durch, wenn er den Prozeß Jesu auf seine eigene Weise darstellt?

Zunächst fällt auf, daß die Erzählung von einer durchgehenden Methodik

1 Vortrag gehalten an der Tagung der S.N.T.S. in Gwatt am 3o. August 1967. 
bestimmt ist. Der Hohe Rat wird nicht als unbefangenes Richterkollegium, sondern als eine voreingenommene Partei geschildert, die mit untauglichen und - was viel schwerer wiegt - mit rechtsverletzenden Mitteln ihr Ziel, die Schuld Jesu festzustellen, zu erreichen versucht. 'Die Hohenpriester und das ganze Synedrium suchten Zeugnis wider Jesus, um ihn zu töten' (V. 55). Die auftretenden Zeugen werden als falsche und sich widerprechende gebrandmarkt (V. 56-9). Weiter sind die Verletzungen des pharisäisch-jüdischen Kapitalprozeßrechtes zu nennen, die sich hier so sehr häufen, daß von einem zufälligen Zusammentreffen keine Rede sein kann. Im Mischnatraktat Sanhedrin 4 werden etliche Vorschriften aufgeführt, die zum Schutz des Angeklagten eingehalten werden müssen: ( I) Beim Kapitalprozeß sollen zuerst die Entlastungs-, dann erst die Belastungszeugen verhört werden. (2) Ein Todesprozeß muß zur Tageszeit geführt werden, da sonst einer der Richter einschlafen könnte. (3) Der Freispruch darf innerhalb eines einzigen Tages ausgesprochen, das Todesurteil muß noch eine Nacht lang überschlafen werden. (4) Darum sitzt man auch nicht am Rüsttag für einen Sabbat oder Feiertag zu Gericht. (5) Die Abgabe der richterlichen Voten hat beim jüngsten Mitglied zu beginnen, damit keiner durch das Gewicht eines älteren Kollegen beeinflußt wird. (6) Nach Sanh. 7, 5 ist der Lästerer erst des Todes schuldig, wenn er den Gottesnamen deutlich ausgesprochen hat. Wir können die Frage, ob diese Rechtsvorschriften zur Zeit Jesu schon in Kraft gestanden haben, auf sich beruhen lassen. Mit Bestimmtheit ist aber anzunehmen, daß diese Grundsätze zu der Zeit, da Markus schreibt, allgemein bekannt sind und Markus deshalb bewußte Anspielungen vornimmt. Damit stellt sich aber mit aller Schärfe die Frage, was er damit beabsichtigt. Will er einfach unterstreichen, daß hier nicht nur nach dem Inhalt, sondern auch der Form nach das Recht verletzt wird?

An dieser Stelle ist ein deutlicher Unterschied vorzunehmen zwischen der einen Frage, ob und wie die Zeugen und Richter zu ihrem Vorgehen legitimiert sind und der anderen, ob die Verurteilung Jesu als solche nicht doch in einem transparenten Sinne legitimer ist, als die illegitimen Richter und Zeugen selbst ahnen. Wie steht es um die Wahrheit der Anklage der falschen Zeugen, daß Jesus beabsichtige, an die Stelle des steinernen Tempels innerhalb von drei Tagen einen zu setzen, der nicht von Menschenhänden gemacht ist (V. $\left.5^{8}\right)$ ? Wieder ist nicht nach der historischen Herkunft dieses Wortes zu fragen, das nach Mark. xv. 29 in der Todesstunde Jesu noch einmal aufgebracht und nach Joh. ii. I9-2 I bewußt auf den Neubau der christlichen Gemeinde bezogen wird. Was würde Jesus, wenn er sich entschlösse zu reden, auf diese Anklage antworten? Markus zielt darauf hin, daß der Leser sofort erkennnt, wie genau mit diesem Vorwurf die letzte Absicht des Lebenswerkes Jesu getroffen ist. Der falsche Zeuge legt ein zwar pervertiertes, ein illegitimes, aber doch den entscheidenen Punkt der ganzen Verhandlung treffendes Zeugnis ab! 
Nicht anders steht es um die Frage des Hohenpriesters und die dadurch ausgelöste Verurteilung Jesu (V. 6I-4). Wieder stellen wir nur beiläufig fest, daß Frage und Antwort sich des Vokabulariums und der Titulatur bedienen, die erst in der nachösterlichen Gemeinde entstanden sind. Dies weiß doch wohl auch der Evangelist. Aber seine Darstellung provoziert noch eine andere Frage: Ist denn Jesus, der sich dazu bekennt, der entscheidende Beauftragte Gottes zu sein, zu Recht oder Unrecht verurteilt worden? Noch mehr. Hier wird der Protest des Lesers provoziert, der sich an die Seite Jesu stellt und bekennt: 'Und er ist es doch!' So ungehörig das Verfahren des Hohenpriesters ist, so trifft es doch den entscheidenden Punkt der Gestalt Jesu. Sie, die Rechtsverletzer, wissen nicht, daß sie ungeahnt mehr Recht haben als ihnen bewußt ist.

Nun ist aber zu beachten, was die von Markus provozierte Reaktion des Leser mit dem Rufe 'Und er ist es doch!' in sich schließt. Wer solche Antwort gibt, ist aus seiner eigenen Neutralität herausgetreten. Er hat sich auf die Möglichkeit, ja Behauptung eingelassen, daß im Prozeß Jesu in ganz anderer Weise, als die Beteiligten wahr haben wollen, Recht gesprochen wird. Denn dann und nur dann ist dieser Protest gegen das illegitime Vorgehen der Richter eine in sich widerspruchslose Sache. Damit tritt aber die kerygmatische Absicht des Markus zu Tage. Er verweist unübersehbar auf ein Geschehen, das sich - die Beteiligten über ihr eigenes Verstehen hinaus in seinen Dienst ziehend - gleichsam hintergründig zu dem sich im Vordergrund vollziehenden Ereignis abspielt. Von diesem Geschehen ist nun aber nicht zu reden, ohne daß zugleich von Gott geredet wird, der in einzigartig legitimer Weise sein Urteil formt und es durch unberufenen Mund aussprechen und dann auch durch unberufene Hände ausführen läßt. Indem Markus den Prozeß als Vollzug einer 'summa iniuria' darstellt, weist er auf jenes Geschehen hin, das sich, den Beteiligten nicht bewußt, als 'summa iustitia' vollzieht.

Nun hält Markus aber bei seiner Darstellung eine bestimmte Grenze sehr genau ein. Er sagt nicht explizit, daß die falschen Zeugen ein letzten Endes zutreffendes Zeugnis über Jesus ablegen oder daß die Verurteilung Jesu zu Recht geschehen sei. Aber durch die Tatsache, daß er erzählt und noch mehr durch die Form, in der er erzählt, schiebt er dem Leser nicht nur neutraliter die Urteilsbildung $z u$, sondern mutet ihm zugleich zu, den Prozeß Jesu als einen von Gott selbst als dem geheimen und zugleich gerechten Richter durchgeführten anzuerkennen. Er provoziert den Leser, von den illegitimen Handlungen weg und auf das Geschehen hinzublicken, das sich nur zwischen Gott und Jesus allein, also in einem besonderen Sinne extra homines abspielt. Damit erhebt Markus aber den Anspruch, daß der historische Prozeß Jesu noch gar nicht durchschaut wäre, wenn auch alle historischen Fragen zur Klärung gekommen wären. Hier weiß Markus um die unüberschreitbare Grenze, die aller historisch-kritischen Forschung um die 
Gestalt Jesu gesetzt ist, gesetzt nicht durch die Unvollkommenheit des historischen Materiales oder der historischen Methode, sondern gezogen durch die Sache selbst, die sich dem rein historischen Zugriff entzieht. Hier wird auch die grundsätzliche Fragwürdigkeit eines jeden Unternehmens enthüllt, das durch historische Rekonstruktion einer Vita oder auch einer geschichtsmächtigen Person Jesu das Geheimnis dieses Mannes zu lüften versucht.

Nun ist die Kontrollfrage zu stellen, ob das, was wir anhand der Verurteilungsszene als Methode des Markus erhoben, auch wirklich zu ihm gehört. Zunächst ist festzustellen, daß weite Teile der Passionsgeschichte die gleiche Darstellungsweise zeigen. Überall stößt man auf gleichsam illegitime Zeugen, die das Entscheidende an Jesus erkennen und aussagen, während andere, die es wissen und sagen sollten, darüber schweigen. Die Frau, die Jesum salbt (xiv. 3-9), weiß nicht was sie tut, während die Jünger, die es erkennen könnten, protestierend daneben stehen. Die Spötter am Kreuze interpretieren wider ihren eigenen Willen den sich vollziehenden Tod Jesu als die Erfüllung des Wortes vom Tempelbau, das viel wahrer ist als sie es wissen (xv. 29 f.). Weder Pilatus noch die Spötter noch auch der Hauptmann des Hinrichtungskommandos wissen, wie viel Wahrheit sie mit der Titulierung Jesu als des Königs der Juden und des Gottessohnes aussprechen (xv. 26, 32, 39).

Das gleiche Mittel wird auch außerhalb der Passionsgeschichte zur Darstellung des Geheimnisses Jesu verwendet. Wie vor allem Eduard Schweizer ${ }^{\circledR}$ in seinem neuen Markuskommentar zeigt, stehen Jesu Nächste und Fernste gleichsam Wand an Wand, indem die einen um das Geheimnis Jesu nichts wissen und doch haarscharf davon reden, während die Nahestehenden völlig blind sind. So stehen sich z. B. in den ersten Kapiteln die Dämonen mit ihrem Christusbekenntnis und die Führer Israels mit ihrem blinden Haß gegenüber, später die Gegner Jesu mit ihrem treffsicheren Blick und die Jünger mit ihrem Mißverständnis. In gleicher weise korrespondieren die Juden mit der syrophönizischen Frau in Kapitel 7 oder die verblendeten Zebedaidensöhne mit dem sehend gewordenen Bartimäus (x. 35 ff., 46 ff.). In diesem Zusammenhang ist die Verschachtelung verschiedener Perikopen zu nennen, ein Verfahren, das Markus wohl nicht selbst geschaffen, aber im Dienste seiner Theologie entscheidend ausgebaut hat. Typisch markinisch ist z. B. die Verflechtung des Vorwurfes der Familienangehörigen, Jesus sei verrückt (iii. 20 f., 3I ff.) mit dem Vorwurf der Schriftgelehrten, Jesus sei teuflischen Geistes (iii. 22-30). Die Antwort Jesu in V. 34-5, daß der zu ihm gehört, der den Willen des Vaters tut, trifft sowohl die nächsten Angehörigen, die in ihrer Harmlosigkeit himmelweit von ihm entfernt sind, wie auch die Gegner, die trotz der Verdrehtheit ihrer Vorwürfe genau gemerkt

\footnotetext{
1 Eduard Schweizer, 'Das Evangelium nach Markus', N.T.D. (1967); zu vergleichen sind z. B. die Überschriften zu i. 14 ; iii. 7 ; vi. $6 b$.
} 
haben, wessen sie sich von ihm her zu versehen haben. Ähnliches ist zu sagen von der Verzahnung des Urteils Jesu über den verdorrten Feigenbaum mit demjenigen über den die echten Früchte versagenden Tempel (xi. 1 2-25), oder von der Verschachtelung des echten Selbstzeugnisses Jesu vor dem Hohen Rat mit der Verleugnung des Petrus (xiv. 54-72). Die ausgewählten Beispiele dürften zur Genüge zeigen, daß solche Methode von Markus als dem Redaktor benützt wird, der teils durch Übernahme älteren Gutes, teils aber durch eigene Neuakzentuierung das Geheimnis Jesu in gleichsam verfremdeter Form darstellt.

So können wir zusammenfassen: Markus bezeugt durch seine transparente und kerygmatische Erzählungsform, daß der Schlüssel zum Verständnis des Prozesses und damit zum Werk und zur Person Jesu in dem Handeln Gottes liegt, der unter der mißverständlichen Form des gebeugten Rechtes sein eigenes Recht durchführt. Indem Markus solches aber nur indirekt ausspricht, schiebt er dem Leser das Urteil über diese Vorgänge als dessen eigenes Glaubensurteil zu.

\section{DER INHALTDES MESSIASGEHEIMNISSES}

Was ist es nun aber um den Inhalt des Messiasgeheimnisses und um den Inhalt der damit provozierten Glaubensentscheidung? Wieder ist bei der Verurteilung Jesu vor dem Hohen Rat einzusetzen.

Mehr noch als die Aussagen der anderen Personen besitzen die Worte Jesu, vor allem sein entscheidendes Bekenntnis ' $k \gamma \omega$ ' $\varepsilon l \mu \mathrm{l}$ ', einen eigentümlichen Tiefgang. Mark. xiv. 62 ist die einzige Stelle im ganzen Evangelium, an der Jesus offen ausspricht, wer und was er ist. Markus stellt Jesus schon zu Beginn der Passionsgeschichte als den um seinen Weg hell Wissenden dar, so z. B. bei der Salbung durch die unbekannte Frau (xiv. 3-9). Was ist nun aber der Inhalt dieses غ̇ $\gamma \omega \dot{~ \varepsilon i ̉ \mu l, ~ w e n n ~ J e s u s ~ d i e ~ v o n ~ s e i n e n ~ u n g e r e c h t e n ~}$ Richtern gestellte Fangfrage durchschaut und dennoch mit einem eindeutigen Ja beantwortet? Sein eigenes Ja richtet sich nun nicht mehr nach der verzerrten Begrifflichkeit der unwissenden Akteure, sondern danach, daß für ihn als den allein Wissenden das laufende Gericht zugleich das Forum Gottes ist. Indem er unter diesem Aspekt seine Antwort abgibt, vollzieht er coram deo die entscheidende Weichenstellung, sein eigens Todesurteil als unter dem Anspruch des göttlichen סєĩ stehend anzunehmen.

Dadurch wird der Inhalt dieses Geschehens in neuer Weise bestimmt. Indem Jesus sich zu den ihm vorgelegten Titeln des Messias und des Gottessohnes bekennt, bekennt er zugleich, daß mit diesen Titeln, sollen sie legitim geführt werden, die Selbstpreisgabe in einen zwielichtigen Tod hinein ver-

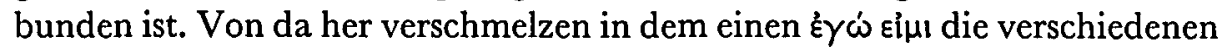
Komponenten der Antwort in eine unauflösliche Einheit: Einmal das Ja zur Úbernahme der vorgelegten Titel, sodann die damit verbundene 
Selbsthingabe und endlich beides als die bejahende Antwort auf eine Forderung des paradoxerweise hinter dem Hohenpriester stehenden Gottes. Dadurch wird nun der Inhalt der verwendeten Titel, die Markus aus der christlichen Tradition entnommen hat, entscheidend bestimmt. Es mag offen bleiben, in welchem Sinne z. B. der Gottessohntitel in der vormarkinischen Tradition verwendet worden ist. Dafür mögen Vorstellungen vom wunder-

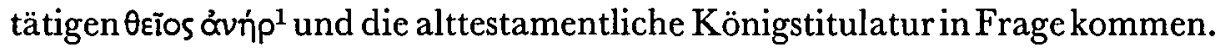
Es mag auch offen bleiben, ob die Markusstellen in den vorangehenden Kapiteln (i. I varia lectio; i. I I ; iii. I I ; v. 7; ix. 7; xiii. 32) mehr nach der einen oder anderen Seite hin tendieren. Durch das Selbstbekenntnis Jesu zu diesem Titel im entscheidungsträchtigen Augenblick wird der Leidensgehorsam zu der allein ausschlaggebenden Komponente der inhaltlichen Füllung. Dies gilt nicht nur für den Messias- und Gottessohntitel. Wenn sich Jesus als der, der sich zum Tode verurteilen läßt, mit dem kommenden Weltenrichter identifiziert, dessen Jurisdiktion alle zukünftigen Räume und Zeiten unterworfen sind, so wird dies unter den Aspekt gerückt, daß Gott selbst es ist, der keinen anderen als den Leidenden dazu bestimmt hat. Die Person des sich zu seiner eigenen Verurteilung bekennenden Jesus füllt die Begriffe und nicht umgekehrt.

An dieser Stelle bricht die alte Frage neu auf, ob Markus bei solcher Gesamtkonzeption seines Evangeliums auf den leidenden Gottesknecht von Deuterojesaja, vor allem Jes. liii anspielt. Unter dem Gesichtspunkt, daß Markus methodisch seine Aussagen bewußt untertreibt und die Schlußfolgerungen aus seiner Darstellung dem Leser zuschiebt, muß ich diese Frage heute, wenn auch in etwas alterierter Form, noch eindeutiger bejahen als vor etlichen Jahren. ${ }^{2}$ Ich fasse meine Anschauung in folgende Punkte zusammen:

(I) Die Verurteilungsszene (xiv. 6I-4) spricht, dies sei ausdrücklich festgestellt, nicht expressis verbis vom stellvertretend sühnenden Gottesknecht. Dagegen wird in konzentriertester Form der konkrete Vollzug des Leidens dessen erzählt, der als der Leidende zum Weltenrichter bestimmt ist. Für Jesus konzentriert sich dieses Leiden in dem frei ausgesprochenen

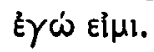

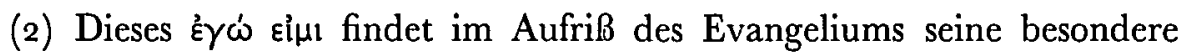
Interpretation durch Texte, die sich auf die Verurteilung Jesu und auf die Vollstreckung dieses Urteils beziehen. Diese Texte erweisen sich innerhalb des Evangeliums als eindeutige Höhepunkte. Zunächst sind die Abendmahlsworte zu nennen, welche durch das Stichwort ékXuvoóuદvov útrè $\pi \circ \lambda \lambda \tilde{\omega} \nu$ (xiv. 24) eine Deutung des Todes Jesu geben, die sich stark an Jes. liii. I I f. anlehnt. Sodann sind die Leidensankündigungen anzuführen,

1 Doch vergleiche die Bedenken dazu bei Wülfing von Martitz und Ed. Schweizer, Artikel viós Th.W. viri, 337 ff.; 354 ff.; bes. $357,16 \mathrm{ff}$.

2 'Knecht Gottes und Sohn Gottes im Passionsbericht des Markus', Z.Th.K. 50 (1953), I-53. 
denen nicht nur die Funktion der Weissagung, sondern ebenso sehr die der

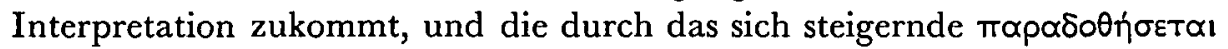
mit Jes. liii. 12 verbunden sind. Vor allem aber besitzt das Wort vom Lösegeld (x. 45) einen eindeutigen Bezug zu Jes. liii. Io. Dieser Spruch, auf dem Wege nach Jerusalem gesprochen, verklammert die galiläische Wirksamkeit Jesu mit der anhebenden Passion und überbietet inhaltlich die vorauslaufenden Leidensankündigungen im Sinne des Sühnetodes.

(3) Nun wird von verschiedener Seite her bestritten, daß die genannten Stellen auf Jes. liii anspielen und erst recht, daß dadurch das Werk und die Person Jesu interpretiert werden. Die Einwände beruhen vor allem auf zwei Voraussetzungen. Zunächst pocht man darauf, daß zwischen Markus und dem Septuagintatext keine Übereinstimmung zu finden sei. Dabei setzt man also voraus, daß die Septuaginta die Grundlage für Markus darstelle. Dagegen ist zu bedenken, daß der Gedanke an den stellvertretenden Sühnetod Jesu schon in der älteren palästinischen Gemeinde lebt. Dafür zeugen nicht nur das alte Bekenntnis I. Kor. xv. 3 f., sondern auch das vorpaulinische Traditionsstück Röm. iv. 24 f. und nicht zuletzt das tief in der Abendmahlstradition sitzende ن்mè $\pi 0 \lambda \lambda \tilde{\omega} \nu$ Mark. xiv. 24 und ähnlich I. Kor. xi. 24. Darum darf man, auch wenn man nicht ins Leben Jesu zurückgreifen will, mit guten Gründen annehmen, das sich das Sühnemotiv schon zu einer Zeit mit Jes. liii verbindet, da die Christen sich noch stärker an die hebräische Bibel und noch nicht an die Septuaginta hielten. Dies ist aber jene frühe Zeit, da sowohl der theologischen Bekenntnisbildung wie auch dem Übergang zum griechischen Sprachraum etwas Tastendes anhaftet. Darum hat bei der Analyse der auf Markus zulaufenden Tradition die Methode der Motivforschung eindeutig den Vorrang vor reiner Wörtervergleichung mit dem Text der Septuaginta. Dies gilt zunächst für die

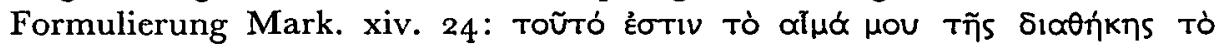

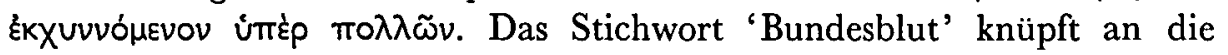
Tradition von Ex. xxiv. 8 an. Der angefügte Partizipialsatz fügt in knappster Formulierung die beiden Sachmotive hinzu, die sich im hebräischen Text von Jes. liii. 12 in enger Verbindung finden: Das Ausschütten, Hingeben des

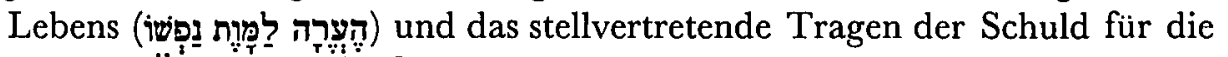

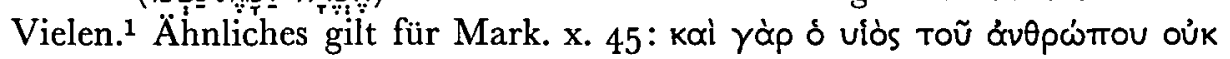

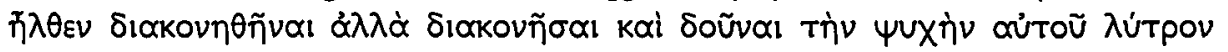

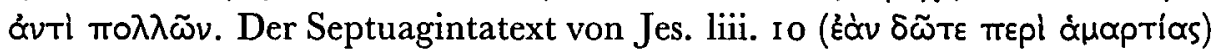
verrät nicht, daß der hebräische Text das Nomen enthält, weshalb ein reiner Wörtervergleich zwischen der Septuaginta und Markus dazu

${ }^{1}$ Ferd. Hahn, Christologische Hoheitstitel, ihre Geschichte im frühen Urchristentum $\left({ }^{2} \mathrm{Ig} 64\right)$, S. $59 \mathrm{ff}$,, fragt mit Recht nach den in der Abendmahlsparadosis enthaltenen Sachmotiven und greift deshalb auf Jes. liii. 12 zurück, ohne sich an der fehlenden wörtlichen Übereinstimmung zu stoßen. Morna D. Hooker, Jesus and the Servant, the influence of the Servant concept of Deutero-Isaiah in the New Testament (1959), S. 80 ff., gelangt zu einem negativen Ergebnis, nicht zuletzt aber deshalb, weil sie nach der Übereinstimmung der einzelnen Wörter sucht. 
führen kann, die beiden Texte als nicht miteinander zusammenhängend zu erklären. Setzt man aber den hebräischen Text voraus, so nimmt Mark. x. $45^{b}$ den Text von. Jes. liii. 10 auf: Ex. Entscheidend ist das Motiv des Loskaufes, das schon Jes. liii. Io nicht im rein kultischen Sinn des Schuldopfers, sondern, weil auf eine Person bezogen, im Sinne einer Ersatzleistung zu verstehen ist, was denn auch in der freien Übersetzung durch das griechische $\lambda \dot{T}$ Tpov zum Ausdruck gebracht wird. ${ }^{1}$

(4) Der zweite Einwand gegen eine bei Markus vorliegende Theologie vom stellvertretenden Gottesknecht betrifft die exegetische Methode des Evangelisten. Es wird betont, daß Markus die atomistische Schriftverwendung der jüdischen Umwelt benütze, die stichwortmäßig eine Einzelstelle aufnimmt, ohne den jeweiligen Gesamtzusammenhang zu berücksichtigen. Darum sei Markus ebenfalls überinterpretiert, wenn man seine Zitate als knappe, aber sehr genau gezielte Hinweise auffasse. Hier bricht eine Kontroverse auf, die in die letzten Tiefen nicht nur der Markus-, sondern auch der Paulusexegese hinein führt. Im Wissen um diese Konsequenzen möchte ich hier an dem einen festhalten: Wenn wir in unserem ersten Teil die untertreibende kerygmatische Methode des Markus richtig erkannt haben, dann dürfen und müssen wir bei ihm eine entsprechende Verwendung alttestamentlicher Stellen annehmen. Dann lebt Markus, weit mehr als die spärlichen Zitate es verraten, in den Gedanken von Deuterojesaja und stellt in bewußter Zielstrebigkeit das Werk und die Person Jesu im Sinne der Erlösung der Vielen durch den stellvertretend leidenden Gottesknecht dar. Dann sind die Abendmahlsworte und der Lytronspruch nicht als blind übernommenes Traditionsgut zu werten, sondern stellen die bewußt aufgesteckten Lichter dar, die zur Erkenntnis des Gesamtskopus des ganzen Evangeliums dienen. Dann aber dürfen auch an anderen Stellen, ohne daß dies einer Textvergewaltigung gleichkommt, die Anspielungen an die Gottesknechtslieder Deuterojesajas als der Absicht des Markus entsprungen verstanden werden. Dies gilt vor allem von jenem makabern Verspottungsspiel (xiv. 65), dem sich die Glieder des Hohen Rates hingeben und das, wie E. Lohmeyer ${ }^{2}$ zu Recht gezeigt hat, eine parodistische Anspielung an Jes. 1. 6 und das dazu gehörige Gottesknechtslied darstellt. Dann ist auch das Schweigen Jesu vor dem Synedrium (xiv. 6I) und vor Pilatus (xv. 5) als

1 C. K. Barrett, The Background of Mark. 10:45 (New Testament Essays, Studies in memory of Thomas Walter Manson, ed. A. J. B. Higgins, 1959, 1-18); Morna D. Hooker a. a. O., S. 74-9 und in ähnlicher Weise auch A. Suhl, Die Funktion der alttestamentlichen Zitate und Anspielungen im Markusevangelium (1965), S. 1 14-20, lehnen den Zusammenhang zwischen Mark. x. $45^{b}$ und Jes. liii. 1o ab. Die vorgenomme Begründung stützt sich weitgehend auf die Vergleichung der Einzelwörter zwischen Markus und dem Septuagintatext statt auf die Gegenüberstellung der Motive.-Ferd. Hahn hingegen (a.a. O., S. 57 ff.) argumentiert mit Recht gegen Barrett vom Inhalt der Aussagen her und schließt sich im wesentlichen den grundlegenden Arbeiten von J. Jeremias ('Das Lösegeld für Viele (Mark. Io, 45)', fudaica, 3 (1947/8), 249-64 (=Abba, 1966, 216-29); ders., Th.W. v, 709 (=Abba 209) und E. Lohse, 'Märtyrer und Gottesknecht, Untersuchungen zur urchristlichen Verkündigung von Sühneleiden Jesu', F.R.L.A.N.T., N.F. 46 ( (1955), i 16 ff.) an.

${ }^{2}$ E. Lohmeyer, 'Das Evangelium des Markus', KEK. (121953) z. St. 
bewußte Aufnahme von Jes. liii. 7 zu verstehen. Und endlich rückt - diesmal in Zusammenhang mit dem Septuagintatext Jes. liii. $6 b$, I2 $b$, I2 $c$, und

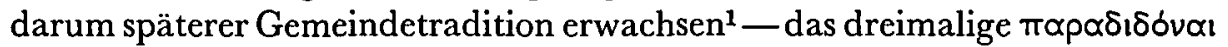
der Leidensweissagungen Mark. ix. 31 ; x. 33 an seine hervorragende Stelle. Wird doch vor allem durch das passivische $\pi \alpha \rho \alpha \delta 0 \theta$ jंбetal das Ineinander des legitimen göttlichen und des illegitimen menschlichen Handelns in seiner ganzen paradoxen Weise zum Ausdruck gebracht.

(5) Nun ist zugleich neu zu überdenken, daß die Gottesstimme bei der Taufe (i. II) und der Verklärung (ix. 7) mitsamt den begleitenden Motiven vollständig aus der Berufung des Gottesknechtes Jes. xlii. I, und zwar unter Wegfall von Ps. ii. 7 erklärt werden kann. ${ }^{2}$ Einzig die Verwendung von viós statt maĩs spricht gegen Jes. xlii. I und für Ps. ii. 7. Hier ist nun zwar leise, aber doch nicht überhörbar zu fragen, ob sich hinter der Gottessohntitulatur bei Markus nicht ganz einfach der Gottesknecht von Deuterojesaja verbirgt. Es gibt ja eine jüdische Tradition griechischer Zunge, welche die beiden Bezeichnungen maĩs und viós ohne jeden Unterschied nebeneinander stellt. Diese wird sichtbar in Sap. ii. I3-20, wo unter Verwendung der deuterojesajanischen Motive der verhöhnte und auf die Zerreißprobe gestellte Ebed Jahve promiscue mit beiden Ausdrücken bezeichnet wird. Wenn Markus sich auf dieser Linie bewegen würde, wäre auch schon das Fehlen des Stichwortes $\pi \propto$ iٓ bei ihm erklärt. Denn dann läge der Tatbestand vor, daß Markus seine entscheidende Aussage, daß der kommende Menschensohn mit dem gekommenen stellvertretend leidenden Gottesknecht eins ist, durch die

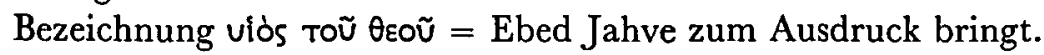

(6) Doch auch dann, wenn das zuletzt Gesagte als rein hypothetisch abzutun wäre, bleibt die sachliche Bedeutung des stellvertretenden Gottesknechtes für die Theologie des Markus bestehen. Man muß sich nur klar machen, daß die Entscheidung primär daran fällt, daß Jesus durch sein

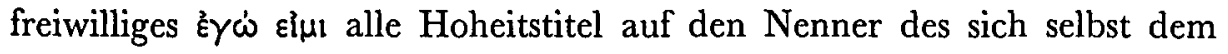
Leiden zur Verfügung stellenden Gottesbeauftragten bringt. Die Gottesknechtsfrage hängt auf das engste mit dem Geheimnis des leidenden Messias zusammen. Davon ist, wenn wir uns nun wieder der Verurteilung Jesu zuwenden, noch ein Letztes zu sagen.

(7) Wenn die entscheidende Realität der so spukhaften Verhörszene darin liegt, daß Jesus sich in seiner Antwort, die zugleich die Selbsthingabe in sich schließt, als der Leidende bekennt, dann fällt noch einmal ein letztes Licht auf die an Jesus handelnden Personen. Es bleibt jetzt nicht mehr bei dem Gleichgewicht der so gegensätzlichen Gestalten: hie Jesus, hie die versagen-

1 Vgl. Ferd. Hahn. a. a. O., S. 62 f.

2 Vgl. vor allem J. Jeremias, Artikel mais, Th.W. v, 699, 3 ff. (= Abba 192 ff.); O. Cullimann, Die Tauflehre des Neuen Testamentes ( $\left.{ }^{2} 195^{8}\right)$, S. I I-13. - Die sprachlichen und sachlichen Gründe, die z. B. Ed. Schweizer, Artikel ulós, Th.W. vill, 369, 7 ff., für die Ableitung von Ps. ii. 7 vorbringt, schrumpfen erheblich zusammen, wenn man ein direktes Zurückgreifen auf den hebräischen Text von Jes. xlii. 1 ins Auge faßt und zudem Sap. ii. $13^{-20}$ berücksichtigt (vgl. oben im Text). 
den Richter und Zeugen. Jetzt erhält das Wort vom kommenden Weltenrichter, das in der Regel als eindeutiges Drohwort aufgefaßt wird, einen mindestens ambivalenten Sinn. Jesus steht vor seinen Richtern als derjenige, der sein Richteramt nicht zur Verurteilung anderer, sondern zum eigenen Verurteiltwerden und damit zum Freispruch für die anderen ausübt. Er ist derselbe, der nach der verschachtelten Geschichte von der Heilung des Gichtbrüchigen die ihm zustehende Vollmacht des Weltenrichters dazu ausübt, Sünden nicht zu strafen, sondern zu vergeben (ii. Io). Er stellt somit auch die ihn verurteilenden Richter noch einmal vor die Einladung, in ihm die Realität des Gottes zu erkennen, der nicht gegen, sondern trotz aller Sünde für sie ist. An dieser Stelle wird die Verurteilungsszene zum Zeugnis des Gotteshandelns nicht nur extra homines, sondern auch pro hominibus. Dies gilt nun aber nicht nur den Richtern, sondern gerade auch dem verleugnenden Jünger Petrus gegenüber. Dies hervorzuheben, ist die Absicht bei der Verschachtelung des Selbstbekenntnisses Jesu mit der Verleugnung des Petrus (V. 54, 66-72 und V. 55-65). Was Petrus tut, rückt, wie auf dem Bilde Rembrandts, in das Licht dessen, der nicht nur gegen, sondern für Petrus sein zum Tode führendes Bekenntnis ablegt. Von da her gesehen wird das bittere Weinen des Jüngers nicht nur zum Zeichen der Reue, sondern zum Zeichen des neu verheißenen Anfangs (V. 72). Noch stärker wird auf dieses pro hominibus in der Pilatusszene angespielt, wo allem Mißverständnis des Prokurators zum Trotz der echte König der Juden dem ihm über den Weg laufenden Barabbas das Leben schenkt (xv. 6-15).

So ist zusammenfassend zu sagen: Der Inhalt des Messiasgeheimnisses ist das im Auftrage Gottes freiwillig übernommene Leiden Jesu als des stellvertretenden Gottesknechtes. ${ }^{1} \mathrm{Da}$ dies aber nicht im Sinne einer objektiv auszusprechenden Tatsache dargestellt, sondern dem Leser als dessen eigene Glaubensentscheidung zugemutet wird, schließt solche Glaubensentscheidung zugleich ein das Leben und die Welt einschließendes Ja zum gnädigen Gott in sich. Denn Markus versteht seine Botschaft nicht als neutrales Kerygma, sondern als heilgefülltes Eu-angelion.

\section{DIE OFFENBARUNG DES GEHEIMNISSES}

Woher nimmt Markus selbst die Freiheit, den tiefsten Inhalt des Jesusgeheimnisses zu kennen, das doch nach seiner eigenen Darstellung den direkt Beteiligten verborgen blieb? Woher weiß er, daß Gott selbst in Jesus handelte, extra homines und zugleich pro hominibus?

Die Antwort auf diese Frage hängt, wie allgemein anerkannt wird, mit Ostern zusammen. Ostern ist der Fluchtpunkt, auf den zunächst einmal die

1 Der alte Kommentar von J. Schniewind zum Markusevangelium, N.T.D. $\left({ }^{10}{ }_{1963}\right)$, ist zwar in manchen Fragestellungen überholt. Aber an diesem einen Punkte ist er von den neueren Arbeiten nicht wieder erreicht worden. 
Leidensweissagungen (viii. $3 \mathrm{I}$; ix. $3 \mathrm{I} ; \mathrm{x} .34$ ), aber auch andere Aussagen (ix. 9 f.; xiv. 28 ; xiv. 62) hinweisen.

Auch die Tatsache des Jüngerunverständnisses, und dieses noch viel stärker, weist in dieselbe Richtung. Die drei Gestalten Petrus, Jakobus und Johannes werden als diejenigen bezeichnet, die in besonderer Nähe Jesu stehen dürfen. Sie sind bei der Auferweckung der Jairustochter (v. 37), bei der Verklärung (ix. 2 ff.) und in Gethsemane dabei (xiv. 33). Petrus ist es, der als erster das Christusbekenntnis ablegt (viii. 29) und sich bis zum Hof des Hohenpriesters vorwagt (xiv. 44). Diese drei Jünger, die am nächsten beim Geheimnis Jesu stehen, versagen in ihrer Weise auf das schmählichste. In der entscheidenden Gethsemanestunde schlafen sie (xiv. $37 \mathrm{ff}$.). Petrus ärgert sich beim Anlaß seines Christusbekenntnisses am Leidenswege Jesu (viii. $32 \mathrm{ff}$.) und versagt vor der harmlosen Magd (xiv. 66 ff.). Die Zebedaiden verraten durch ihre Sonderbitte das völlig Mißverständnis der Sendung Jesu (x. 35 ff.).

Es wäre wohl verfehlt, wenn man einen Angriff des Markus auf diese Gestalten annehmen wollte. Vielmehr stellt sich wieder die Frage nach der Absicht dieser Darstellung im Sinne der Methode des Evangelisten. Einzusetzen ist bei der Feststellung, was denn die Leser des Markus von diesen drei Gestalten wissen. Petrus und Jakobus sind in den Sechzigerjahren bereits als bestandene Blutzeugen bekannt. Von Johannes darf ein gleiches aus dem vaticinium ex eventu (x. 39) mit großer Wahrscheinlichkeit erschlossen werden. Sie, die das Leidensgeheimnis zu Lebezeiten Jesu nicht erkannten, haben dieses später durch ihre ganze Existenz bezeugt. Da Markus nicht am Reifeprozeß eines Menschen interessiert ist, dienen ihm die drei Sonderjünger dazu, den Qualitätsunterschied der Zeit vor und nach Ostern zu kennzeichnen. Bevor Jesus seinen Weg zum Kreuz vollendet hatte, konnten sein Werk und seine Person ambivalent als zur Hoheit oder zur Niedrigkeit führend verstanden werden. Durch die Vollendung seines Gehorsams in der Niedrigkeit des Kreuzes stellte sich erst recht die Frage, ob solches Geschehen von Gottes Absicht her und darum auch als Heil verstanden werden dürfe. Die Antwort kam erst durch das Ostergeschehen. Hier erhielt das Messiasgeheimnis als das Leidensgeheimnis jene Durchschlagskraft und Vollmacht, die - unter dem eschatologischen Vorbehalt - vom Jüngermißverständnis zur vollen Erkenntnis und damit zu einer auch das Leiden einschließenden Nachfolge führen konnte. Erst nachträglich, wo auch das vorauslaufende prae homines des Christusgeschehens eingesehen werden konnte, enthüllte sich das Handeln Gottes in seiner ganzen Tiefe als ein Handeln extra homines und pro hominibus.

Wir gehen nicht auf die quälenden Rätsel ein, die der so brüsk abbrechende Markustext stellt. Immerhin hat Markus auf jeden Fall mit Erscheinungen des Auferstandenen vor seinen Jüngern gerechnet. Es ist historisch nicht mehr auszumachen, wie er die Erscheinungen Jesu, und übrigens auch das leere 
Grab im einzelnen verstanden hat. Aber aus dem Gesamtduktus seines Evangeliums ist zu schließen, daß es ihm um einen radikaleren Einbruch des gnädigen und realen Gottes in diese Schöpfung herein geht, als alles menschliche Verstehen erfassen kann. Wenn solches auch nur in aller Unvollkommenheit gilt, dann treiben wir auch heute noch, allem Unverstand und Mißverständnis, denen gerade wir Theologen ausgesetzt sind, zum Trotz, unser eigenes Werk nicht ohne Verheißung.

CHRISTIAN MAURER

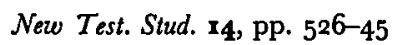

\section{SOME DISTINGTIVE EARLY GHRISTOLOGIGAL MOTIFS}

Too often the Christology of early Palestinian Christianity has been determined by (I) evaluating the Hellenistic contribution to the New Testament, subtracting this from the total, and acclaiming the residue to be Semitic and primitive (oblivious to the intermingling of Hebraic and Hellenistic ideologies in first-century Palestine), and (2) working from a predetermined set of convictions as to what was possible in a Hebraic milieu. Now a prioris can never be eliminated altogether from any study; and certainly should not be condemned per se, for without them one would never begin. But they must be checked both historically and critically.

In large measure this process of 'Hellenistic subtraction' in the past has been abetted by the nature and paucity of extant materials with which the New Testament could be compared. But with the discoveries of the Dead Sea and Nag Hammadi texts, and the resultant re-evaluation of previously known non-canonical writings, material is now at hand for a new application of the old comparative-religion methodology which gives promise of more adequately based results.

It is the thesis of this paper that certain portions of the New Testament stand in direct ideological and conceptual continuity with the Dead Sea Scrolls and related Old Testament apocryphal literature, on the one hand, and the post-apostolic Jewish Christian writings and related Nag Hammadi texts, on the other; and that by the tracing of parallels of imagery and expression between these three bodies of material-with appropriate allowances for differences of theological outlook and the development of thought-there result some interesting identifications and conclusions regarding early Palestinian Christology such as were not possible before. Following out the suggestions and work of others, it is my purpose here to isolate some dis- 